\title{
Building Bridges with the Right Tools: Modality and the Standard Model
}

\author{
Steven French \\ School of PRHS \\ University of Leeds \\ Leeds, UK
}

\begin{abstract}
The current state of the relationship between metaphysics and the philosophy of science might appear to be one best described as 'hostility on both sides'. In an attempt to bridge this gap, French and McKenzie (2013) have suggested a two fold strategy: on the one hand, if metaphysics is to be taken to have something direct to say about reality, the implications of physics need to be properly appreciated; on the other, one does not have to agree with the claim that $a$ prioristic metaphysics should be dismissed or even discontinued, since we should value scientifically disinterested metaphysics as a 'toolbox' for philosophers of science. It is in the context of this strategy that I want to approach the issue of understanding the symmetry principles that feature in the Standard Model of modern physics. I shall suggest that the dispositional analysis of laws is incapable of accommodating such principles. However, there are other tools in the metaphysical toolbox that one can draw upon to help capture the nature of such symmetries corresponding to the second part of the above strategy.
\end{abstract}

\section{Introduction}

The current state of the relationship between metaphysics and the philosophy of science might appear to be one best described as 'hostility on both sides'. Certain philosophers of science have dismissed much of current metaphysics as disconnected from the implications of modern science or based on high-school chemistry at best. Some metaphysicians, for their part, have insisted that their aim is to explore the space of the possible and not to be confined to the actual. In an attempt to bridge this gap, French and McKenzie (2013) have suggested a two fold strategy: on the one hand, if metaphysics is to be taken to have something direct to say about reality, the implications of physics need to be properly appreciated and this will have implications for certain 'paradigmatic' metaphysical positions; on the other, one does not have to agree with the claim that a prioristic metaphysics should be dismissed or even discontinued, since various considerations suggest that we should value scientifically disinterested metaphysics as a 'toolbox' for philosophers of science.

It is in the context of this overall strategy that I want to approach the issue of understanding the Standard Model of modern high-energy physics. In particular, my concern is how we should understand the nature and role of the symmetry principles that are crucial features of this model. I shall suggest that the standard dispositional analysis of laws is incapable of accommodating such principles. In effect this is to deploy the first part of the above two-fold strategy, according to which the implications of modern physics may well rule out certain metaphysical positions. However, there are other tools in the metaphysical 
toolbox that one can draw upon to help capture the nature of symmetries in physics, corresponding to the second part of the strategy.

\section{The Death of Dispositionalism}

Dispositionalist and powers based analyses of properties have extended their reach recently, from vases and fragility to electrons and charge. Thus Mumford writes:

'[p]hysics in particular seems to invoke powers, forces and propensities, such as the spin, charge, mass and radioactive decay of subatomic particles' (Mumford, 2011: 267).

The core idea is that such fundamental properties of physics as spin, charge and mass can all be understood in dispositional terms, where that involves the appropriate stimulus yielding the relevant manifestation. Think of charge for example: we take a given charge and bring up a test charge to a certain distance, whereupon a certain force and acceleration is experienced (either towards or from the given charge). The bringing up of the test charge is the stimulus and the force or acceleration experienced is the manifestation.

On the standard view, dispositions can thus be captured in terms of the 'Stimulus and Manifestation Characterisation' (S\&M):

$$
\forall x((\mathrm{P} x \& \mathrm{~S} x) \rightarrow \mathrm{M} x)
$$

where $\mathrm{P}$ is the relevant property, such as charge, $\mathrm{S}$ the stimulus and $\mathrm{M}$ the manifestation.

This then yields the corresponding laws (see Bird 2007) and one of the core advantages of this characterisation is that it explains the physical necessity of those laws: in any possible world that contains objects with property $\mathrm{P}$, the presence of the stimulus will yield the relevant manifestation; hence in those worlds, the corresponding laws will hold.

\section{The Standard Model}

Now, when it comes to quantum physics, the fundamental law is given by Schrödinger's Equation:

$$
i \hbar \frac{\partial \mid \varphi\left(n_{i}\right)>}{\partial t}=H \mid \varphi\left(n_{i}\right)>
$$

where $\mathrm{H}$ denotes a specific Hamiltonian and the $n_{i}$ denote the state-independent properties that identify the kind of particle involved. Of course, as presented thus it is perfectly general and to get something applicable to a particular system, we need to 'plug in' the relevant Hamiltonian. To attribute a property to a particle obeying one of these specific laws, the operator for that property must commute with the corresponding Hamiltonian. Sets of such operators then form a group representing a symmetry of the system. By virtue of the requirement that these operators must commute with the relevant Hamiltonian, the symmetries can be 
viewed as acting as constraints on particular Hamiltonians and their associated specific laws.

What symmetries are we talking about? Well, first of all there is the Permutation Invariance that arises from the so-called indistinguishability of quantum particles (see French and Krause 2006). As a constraint it effectively divides up the Hilbert space into non-combining sectors, each corresponding to a particular irreducible representation of the permutation group and thus to a certain kind of particle and yielding a particular form of quantum statistics, the two most well known being fermions, which obey Fermi-Dirac statistics and bosons, obeying Bose-Einstein statistics.

Secondly, the underlying quantum theory of the Standard Model is, of course, quantum field theory, so the second set of symmetries that needs to be considered are those of Minkowski space-time - the space-time of Special Relativity. These are captured by the Poincaré group and the irreducible representations of this group yield a classification of all elementary particles, with these representations indexed or characterised by mass and spin (the invariants of the group).

Finally, the Standard Model (SM) itself is fundamentally a gauge theory, represented by the group SU(3) x SU(2) x U(1) via which the relevant symmetries can be captured within the theory. That the SM is a gauge theory refers to the way in which the Lagrangian of a system - which basically captures the dynamics of that system - remains invariant under a group of transformations, where the 'gauge' denotes certain redundant degrees of freedom of that Lagrangian. The generator of the group of transformations represents a field and when such a field is quantised, we get the so-called gauge bosons. Thus, consider electrodynamics, for example: the relevant gauge symmetry group associated with the property of charge is labelled U(1) and the gauge boson that effectively drops out of the requirement of gauge invariance is the familiar photon. This requirement can then be extended to the other forces in physics and so, for the weak nuclear force, we have the SU(2) symmetry group associated with isospin, a property of protons and neutrons, and the strong nuclear force associated with SU(3) which operates on the colour property of quarks. With the Higgs boson associated with the breaking of the isospin symmetry of the unified electro-weak force and responsible for the acquisition of mass, we have a complete picture of the fundamental forces, gravity excepting of course.

So, we have the 'internal' gauge symmetries, the 'external' space-time symmetries and Permutation symmetry. And the blunt question for the dispositionalist is: how are you going to accommodate them? Frankly, I do not think she can (for similar sentiments, see Psillos 1996; Lange 2012; and for further discussion in this specific context see Cei and French 2014, French 2014). Consider the S\&M characterisation above: how might this accommodate the afore-mentioned symmetry principles? It does not seem to be the case that it is in response to a certain kind of stimulus that a quantum particle manifests fermionic or bosonic behaviour, or cannot accelerate past the speed of light or has its interactions governed by the SU(3) symmetry, say. Indeed, dispositionalists themselves seem to appreciate the difficulty in which they find themselves, with Bird, for example, suggesting that these symmetries should be dismissed as mere 'pseudo-laws' that will eventually be eliminated from physics. 
One might be tempted to shift to an alternative account, such as the socalled 'Humean' picture. According to this, the underlying metaphysics is that of a 'mosaic' of properties (the so-called 'perfectly natural' ones) instantiated at space-time points. This mosaic exhibits certain regularities and those that we represent via our 'best' system are deemed to be laws (see Cohen and Callender 2009). However, little, if anything, has been said on how the Humean might accommodate the above symmetry principles.

Here a dilemma arises (see Hall 2015) : first, this combination, of the Humean mosaic with its regularities, and a 'best system account' of laws (plus symmetries) just does not mesh nicely with the practice of physics. In particular, that practice makes a clear distinction between what can be called the 'Dynamical Hypothesis' (DH) and the 'Initial Conditions Hypothesis' (ICH), into which the nomologically possible histories of particle behaviour are factored by the fundamental laws (ibid.). Our system will then be better to the extent that the latter is as uninformative as possible, in the sense that it should admit as broad a range of initial conditions as nomologically permitted, whereas the former should be as informative as possible, this combination yielding the maximum explanatory power.

Unfortunately, this distinction is entirely unmotivated by the underlying metaphysics of the Humean mosaic (ibid.). To see this, consider the Humean conception of law: on this view, a claim about the world counts as law-like only if it can be regarded as a 'distinctively appropriate target for scientific inquiry', independent of its nomological status. The question then is, how, from all the various distributions of perfectly natural properties spread across the mosaic, do we pick out those that count as 'distinctively appropriate'? Lewis, famously, argued that we should pick out those that meet certain standards of simplicity and informativeness, but the latter, is precisely not what we want when it comes to the ICH.

Thus, the Humean faces a dilemma: on the one hand, she can ensure that her account meshes with the practice of physics by incorporating the ICH/DH distinction, but then the choice of the resulting standards (for judging what is a law etc.) are entirely unmotivated by the metaphysics of the mosaic; or she can choose standards that are so motivated, but then her account will be out of kilter with scientific practice. As Hall puts it, 'This choice between a guilty intellectual conscience and insane revisionism is not a happy one.' (ibid., p. 51).

Bringing symmetries on stage renders this dilemma even more acute. The practice of physics suggests that symmetry principles should be regarded as further constraints on the $\mathrm{DH}$ : in the relativistic context, for example, only those dynamical hypotheses are allowed that conform to the symmetries represented by the Poincaré group; in the context of quantum mechanics, only those Hamiltonians will be considered that commute with the particle permutation operator. Thus, in addition to those restrictions on the ICH that would allow us, for example, to construct a mathematically acceptable DH to begin with, we have the further restrictions imposed on the DH by the relevant symmetries. Again, we face the issue of how these restrictions might be motivated by the metaphysics of the mosaic.

The Humean could argue that we should consider the nature of physics practice more carefully. Of course, it would be hard to deny that such symmetry principles do play a heuristic role in theory construction but this does not mean 
that they should be understood as constraining the DH in the way that the ICH does. Indeed, the Humean can simply insist that these symmetries reflect metalevel regularities that hold between the law-like regularities across the mosaic. Thus, the Poincaré symmetries are just meta-level features of the laws of Special Relativity and Permutation Symmetry can be understood as a manifestation of the peculiar nature of the entities of which the mosaic is composed (French and Krause 2006).

On this view, then, there are no constraints, strictly speaking, And to suggest that the only alternative is to regard these principles as 'mere' coincidences, would be to miss the point: as far as the Humean is concerned, that the mosaic displays certain regularities and that these regularities display certain similarities themselves, is just a fact of the way the world is. Of course, the accompanying 'best system account' must be further extended to include these symmetries as also part of the 'system' but now Hall's dilemma bites again.

One might think that there is nothing in principle preventing such an extension: those meta-regularities that meet whatever criteria are deployed to evaluate which system is 'the best' are deemed to be the symmetry principles. However, on the very basis just set out, a claim about the world counts as a 'meta-regularity', in this sense, only if it too can be regarded as a 'distinctively appropriate target for scientific inquiry', independently of its meta-law-like status. But what criteria do we use for determining which of the various metaregularities spread across the mosaic are deemed to be 'distinctively appropriate' in this sense? Granted, since we are reconstruing scientific practice in accordance with this response, we don't have to worry about accommodating the way physics views the ICH/DH distinction, and so the Humean might, for example, revert to the kind of traditional criteria that Hall dismissed as failing to mesh with that practice; criteria such as 'informativeness' or explanatory power. But the extent to which symmetry principles might be taken to possess such power is only just being explored and certainly further work needs to be undertaken before pursuing this line.

Hall concludes his analysis by asking what metaphysical viewpoint might motivate the kind of distinction he has set out between the ICH and the DH and suggests that it must be the kind of view that takes information about the counterfactual structure of the world to be sui generis (Hall ibid., p.52). With dispositionalism ruled out by contemporary physics, what other view can meet this demand?

\section{New Tools from the Toolbox}

There are in fact a number of recent analyses that one might deploy here, some of which are extensions or variants of the dispositionalist framework, others of which are quite different (French forthcoming). Here is one such: Vetter's account of potentiality (Vetter 2015).

She begins by noting that the conditional analysis that lies behind the S\&M characterisation of dispositionality lies in tension with our linguistic means by which we ascribe dispositions, via adjectives such as 'fragile', 'irascible', or 'soluble'. As she notes, these all refer to only one half of the putative conditional, namely the relevant manifestation in each case. Furthermore, she argues, the most natural way of paraphrasing for the -le suffix through which they are 
formed is 'can' and other expressions of possibility. This motivates her account, which characterises the relevant modality via a form of possibility.

More particularly, such a possibility is a potentiality of some object, where this is individuated by its manifestation. Thus we drop the stimulus and with it, the S\&M characterisation. The modal nature of the possibility is then best expressed simply by ' $x$ can $M$ ' and the difference with the dispositional account can be seen if we consider the context-dependence and vagueness of terms such as 'fragile'. This context dependence comes down to the degrees of fragility, in the sense that whether something - a vase, say - counts as fragile in a given context has to do with how fragile it is. The dispositionalist will accommodate that dependence in terms of a property that comes in degrees, some of which will be sufficient for the correct application of 'is fragile'. For Vetter, by contrast, being fragile is a matter of having the right potentiality to a contextually sufficient degree (ibid., pp. 21-22).

Of course, we lose the counterfactual analysis underpinning the standard dispositionalist account, and we lose the tight connection with causation. But then, of course, the latter is a problem when it comes to the foundations of physics. We also lose the explanatory value of dispositionalism in accounting for the modal necessity of laws but Vetter argues that this was always illusory, since all that the S\&M characterisation gives us is a conjunction of law instances, rather than the law per se (Vetter 2009).

On her view, it is potentialities 'all the way down', so that the fundamental properties are potentialities, possessed, due to their fundamentality, to the maximum degree (2015, p. 285). Taking classical physics, on this view objects that have charge have the potentiality (to maximal degree) to exert a force whose value is related to that of other charges and their distance from that object through Coulomb's Law (ibid.). This leaves open questions about the modal status of such laws, in the sense that their necessity or otherwise does not simply follow from the modal metaphysics of potentiality (ibid., pp. 288-290). This might seem like a significant loss of metaphysical explanatory power but, again, this was never really there to begin with and as we have seen, cannot be obtained anyway when it comes to the symmetries. So, perhaps we can turn this to our advantage and use Vetter's account to capture the modal aspects of the Standard Model.

Of course, we do not have the equivalent in the language of physics of adjectives such as fragile; we do not (typically) refer to electrons as 'spinnable', for example! However we do have something similar, namely the language of mathematics, in which modern physics is expressed (suitably interpreted of course). It is this that reveals the possibilities inherent in the particular symmetry we might be concerned with. Thus, consider again Permutation Symmetry: if one reads the standard range of textbooks in quantum physics, one will find statements to the effect that the wave functions of quantum systems must be either symmetrical or anti-symmetrical, corresponding to Bose-Einstein and Fermi-Dirac statistics, resepctively. But as was recognised early on, and as was made explicit in the late 1950s and 1960s (for a history of such developments, see French and Krause 2006) , the mathematics of the more general Permutation Invariance contains a whole range of other possible 
statistics including so-called 'paraparticle' and 'infinite' statistics ${ }^{1}$. Likewise, if one considers spaces of dimension less than 3, one can obtain non-standard 'anyon' statistics as described by the 'braid' group (see French 2000) and which play an important role in the explanation of the fractional quantum Hall effect ${ }^{2}$.

And of course, we don't have the 'gradability' or contextuality that are associated with claims such as 'the vase is fragile' but we obviously do have a different range of possibilities afforded by different symmetries: in addition to the above, there is the afore-mentioned range of particle properties, including mass and spin, in the case of Poincaré symmetry. Furthermore, the different possibilities encoded in these principles - e.g as we move from 'standard' quantum statistics to the paraparticle form - correspond to counterfactual scenarios that allow us to account for the explanatory role of such symmetries (see French and Saatsi forthcoming).

Furthermore, the simple Vetter formula for accommodating potentiality via ' $x$ can $M$ ', where $M$ is the manifestation of this potentiality, can also be adapted to this purpose. Take ' $x$ ' to be the relevant symmetry, then the manifestation will be the property of spin, say, in the case of Poincaré symmetry or that of being a boson, fermion, paraparticle or anyon in the case of Permutation Invariance. Eliminating stimuli from this account enables us to at least begin to accommodate the modal features of such principles without having to unreasonably contort the physics. In addition, we can accommodate Vetter's distinction between 'mere' possibility and potentiality in terms of the latter standing to the former as essence stands to necessity, namely as the localized counterpart of a non-localized modality' (Vetter 2014, p. 23). Again take Permutation Invariance: the full range of mathematical possibilities encoded here corresponds to all the representations of the permutation group, but only the symmetric and antisymmetric are manifested in this, the actual, world ${ }^{3}$. That full range of mathematical structures can be viewed as the 'non-localized' possibility and the specific representations that apply to the world can be understood as the localized counterparts.

Of course, this simplistic equation between possibility as abstract and potential as concrete may raise concerns in this specific context, namely that here modality is being reduced to the mathematical; or more acutely, perhaps, that physical possibility is being reduced to mathematical possibility. However, I see this as a virtue rather than a vice! Leaving aside the tricky issue of how we might distinguish the physical from the mathematical, this way of viewing things makes explicit the role of surplus structure in the history of modern physics as a form of drawing upon further possibilities and making manifest the underlying

\footnotetext{
${ }^{1}$ As is now well-known, it was suggested that quarks obey a form of the latter, before it was shown that their statistical behaviour could be equally well represented via the introduction of a further parameter that came to be called 'colour'. The speculation that the choice between applying parastatistics and introducing a new property is in certain respects conventional has been firmed up by Baker et. al. 2015. This might be viewed as adding further heft to the Vetterian approach.

2 There is some debate as to whether anyons should be considered mere mathematical artefacts or 'real' and possibly manipulable entities. In view of what I shall say below, one might question this distinction.

${ }^{3}$ Although if one accepts Baker et. al.'s result that there is a certain conventionality that holds between conceiving of quarks as parafermions of order 3 and as possessing colour, then one might suggest that other representations are in fact manifested in this world as well.
} 
modality. Again, the irreducible representations of the Permutation Group corresponding to parafermionic statistics are just so much surplus structure, from the point of view of the standard bosonic and fermionic forms, but become physically manifest when applied to quark statistics. Ditto the representations of the braid group underpinning anyon statistics.

One might still balk at this apparent shift of the locus of potentiality from objects - as Vetter emphasises - to certain principles, even if the latter are regarded as 'meta-laws', in some sense, with associated notions of (meta) governance. However, one could retain objects as elements of one's metaphysical pantheon and still pack all the relevant potentiality into the symmetries. Thus, one could, for example, give up a substantival understanding of such objects (Paul 2013) and accept them as bundles of properties and, crucially, relations (Chakravartty 2007), where the latter, in turn, are seen as the concrete exemplifications of what we call laws and symmetries - so the idea would be akin to Humeanism in the sense that these laws and symmetries do not govern the behaviour of the objects, in the sense of being imposed upon them, but are simply the descriptions (within our 'best' system) of certain regularities and regularities between regularities that hold across the distribution of objects, their properties and relations. Of course, such relations would still embody the relevant modal features, so in that sense this picture is certainly not Humean in flavour, and one still has to explicitly address the Hall problem outlined above, but this may be one way to go if one wishes to retain objects.

Alternatively one can give them up, either entirely or as anything other than formal or conceptual placeholders, of a delicate metaphysical thinness. That way leads to structuralism, of either an eliminativist or 'moderate' variety, with the modally informed symmetries understood as metaphysically fundamental. In a sense this too offers a third way between dispositionalism and Humeanism as unlike the latter it retains the modal features but unlike the former it shifts metaphysical attention away from putative objects as particulars. However, that has been discussed elsewhere and the considerations presented above can be seen as a further addendum to that discussion (see French 2014, where the focus is on the determinable-determinate distinction as a metaphysical tool used to help explicate the relationship between symmetries and properties).

\section{The Thinness of the Tools}

There are two further concerns that may also arise about this use of metaphysical devices, such as Vetter's notion of potentiality, as tools in this context. The first is that they are too 'thin' or insubstantial to properly serve as tools in this sense, since all the work is effectively done by the relevant physics. Consider again Vetter's idea that modality can be captured via the formula ' $x$ can $M$ '. For this to even begin to get a grip on the job, it needs to be supplemented with $x$ understood as the relevant symmetry and $M$ as the manifestation, whether properties or kinds or whatever. The formula itself and the attendant understanding in terms of localization, contextuality and all the rest, seem to do little, if any, of the actual 'work', however that is understood.

But then what did we expect? Insofar as 'spin' drops out of the Poincaré group or Bose-Einstein statistics from Permutation Invariance, all the deductive work is being doing by the relevant mathematics. Of course it is - how could it be otherwise? First of all, so-called aprioristic metaphysics - that is metaphysics 
that is not already associated with science in some form - is standardly dismissed as doing no 'work' (McLaurin and Dyke 2012). Given that this is the kind of metaphysics that French and McKenzie claim can yet be drawn upon as a tool, it should come as no surprise that, given its non-naturalistic nature, when used as such it has to be supplemented with the relevant physics. Naturalistic or non-analytic metaphysics presumably has the relevant physics - or some other science - already 'built in'.

Secondly, as supplemented, this metaphysics-as-tool nevertheless yields something important, namely a form of (metaphysical) understanding, which may be regarded as a kind of metaphysical unification. Consider again: our core issue was how to understand what appeared to be prima facie, certain modal features of the Standard Model and modern physics more generally. Accommodating these within the standard dispositionalist framework with its S\&M characterisation just isn't an option, bluntly because we can't make sense of that characterisation in this context. Giving up on modality altogether and going Humean also seems a dead end, given Hall's problem. Vetter's potentiality offers us a metaphysical device that we may adapt to our circumstances and by doing so relate them to the 'everyday' circumstances of vases and the like. Of course there are differences but the deployment of such a tool helps to illustrate possible metaphysical similarities, thereby offering the hope of understanding the nature of modality in the context of modern physics by relating it to the way modality works in ordinary language.

Now perhaps, on further analysis, this just won't work. Maybe in that case we have to go au naturel and develop an appropriate naturalistic metaphysics fit for purpose. But designing such a bespoke metaphysics is no easy task and by virtue of its very nature - that is, its not being tied to any metaphysics of the everyday, even in the thin sense as indicated above - may undermine the possibility of understanding. Consider, as a warning, Eddington's last great work, entitled Fundamental Theory, in which he attempted to articulate what was effectively a form of quantum gravity in his own (physical and broadly metaphysical) terms, where those terms prove to be almost impenetrable to everyone else.

\section{Relieving the Tension}

There is a further concern: if such metaphysics does prove useful as a 'tool', do philosophers of science then have any grounds for castigating or admonishing or, generally, wagging their fingers at metaphysicians who appeal to their intuitions, or classical or even Aristotelian, physics or who ignore or pay little attention to science in general? An obvious tension arises between the two sides of the French-McKenzie position (see French and McKenzie 2015). There is, however, a response that eases this tension:

What the afore-mentioned anti-metaphysical philosophers of science are objecting to is the filling in of certain conceptual frameworks with intuitions, classical conceptions or, more generally, an inadequate understanding of physics. That's where point 1) above of the French and McKenzie response bites, But qua frameworks, such devices and moves may still prove useful tools as the example of 'potentiality' suggests. That's what lies behind point 2). The filling in, or supplementing, is then undertaken by the relevant physics, as indicated above. 
The role of metaphysics as a tool is then doubly conditional: first, upon naturalistic metaphysicians and philosophers of science continuing to take metaphysics down 'off the shelf', instead of making metaphysics to order by themselves; and, secondly, upon those analytical constructions actually turning out to be relevant and useful to the interpretation of science as it evolves. In both cases, this will all be dependent on developments not only in science but also in philosophy of science. As things stand however, and at the very least, there remains the possibility (ha ha) of metaphysics remaining useful for modern physics.

\section{References}

Baker, D.J., Halvorson, H. and Swanson, N. 2015. The Conventionality of Parastatistics. British Journal for the Philosophy of Science 66 : 929-976

Bird, A. 2007. Nature's Metaphysics: Laws and Properties Oxford: Oxford University Press

Cei, A. and French, S. 2014. Getting Away from Governance: Laws, Symmetries and Objects. Méthode - Analytic Perspectives 3 doi: http://dx.doi.org/10.13135/2281-0498\%2F4

Chakravartty , A. 2007. The Metaphysics of Science. Cambridge: Cambridge University Press.

Cohen, J. and Callender, C., 2009. A better best system account of lawhood. Philosophical Studies 145:1-34

Eddington, A. 1946. Fundamental Theory. Cambridge: Cambridge University Press.

French, S. 2000. Putting a New Spin on Particle Identity. In Spin-Statistics Connection and Commutation Relations. ed. Hilborn, R. and Tino, G., 305-318, American Institute of Physics.

French, S. 2014. The Structure of the World. Oxford: Oxford University Press.

French, S. forthcoming. Doing Away with Dispositions: Powers in the Context of Modern Physics. In Dispositionalism: Perspectives from Metaphysics and the Philosophy of Science. ed. A. S. Meincke-Spann. Springer Synthese Library, Springer.

French, S. and Krause, D. 2006. Identity in Physics: A Historical, Philosophical , and Formal Analysis. Oxford: Oxford University Press. 
French, S. and McKenzie, K. 2012. Thinking Outside the (Tool)Box: Towards a More Productive Engagement Between Metaphysics and Philosophy of Physics. The European Journal of Analytic Philosophy 8: 42-59

French, S. and McKenzie, K. 2015. Rethinking Outside the Toolbox: Reflecting Again on the Relationship Between Philosophy of Science and Metaphysics. In Metaphysics in Contemporary Physics. ed. T. Bigaj and C. Wuthrich , 145-174, Poznan Studies in the Philosophy of the Sciences and the Humanities, Rodopi.

French, S and Saatsi, J. forthcoming. The Explanatory Role of Symmetries.

Hall, N. 2015. Humean Reductionism about Laws of Nature. In A Companion to David Lewis. eds B. Loewer and J. Schaffer, Oxford: Wiley.

Lange, M. 2012. "There Sweep Great General Principles Which All the Laws Seem to Follow". In Oxford Studies in Metaphysics, volume 7, ed. Karen Bennett and Dean Zimmerman, pp. 154-185, Oxford: Oxford University Press, 2012.

Maclaurin, J. and Dyke, H. 2012. What is Analytic Metaphysics For? Australasian Journal of Philosophy, 90: 291-306

Mumford, S. 2011. Causal Powers and Capacities. In The Oxford Handbook of Causation. ed. H. Beebee, P. Menzies, and C. Hitchcock, pp. 265-278, Oxford: Oxford University Press.

Paul. L.A. 2013. Categorical Priority and Categorical Collapse. Proceedings of the Aristotelian Society 87: 89-113.

Psillos, S. 2006. What do powers do when they are not manifested? Philosophy and Phenomenological Research 72: 135-156.

Vetter, B., 2009. Review of Bird. Logical Analysis and History of Philosophy 8: 320-328

Vetter, B. 2014. Dispositions without Conditionals. Mind 123: 129-156.

Vetter, B. 2015. Potentiality: From Dispositions to Modality. Oxford: Oxford University Press. 